\title{
p

\section{A new hydrogel for the conservative treatment of meniscal lesions: a randomized controlled study}

\author{
CLAUDIO ZORZI ${ }^{1}$, STEFANO RIGOTTI ${ }^{1}$, DANIELE SCREPIS ${ }^{1}$, NICOLA GIORDAN², \\ GIANLUCA PIOVAN ${ }^{1}$ \\ ${ }^{1}$ Department of Orthopaedics, Sacro Cuore Hospital of Negrar, Negrar (VR), Italy \\ 2 Department of Clinical Operations, Fidia Farmaceutici SpA, Abano Terme (PD), Italy
}

\begin{abstract}
Purpose: this study aimed to investigate the efficacy of intra-articular (IA) administration of a hydrogel formulation obtained from a hyaluronic acid (HA) derivative $\left(\mathrm{HYADD} 4^{\circledR}\right)$ in the management of meniscal tears and in meniscal tear repair.

Methods: fifty subjects with degenerative meniscal tears were enrolled into this single-site, observer-blind, parallel-group study. Clinical evaluations were performed at baseline and after 14, 30 and 60 days. Clinical outcomes included: pain reduction (Visual Analog Scale), improvement of knee functionality (WOMAC questionnaire), reduction in length and depth of the meniscal lesion (MRI-confirmed) and SF-36 questionnaire scores. Local tolerability and safety were also investigated.
\end{abstract}

Results: a significant reduction in VAS pain $(\mathrm{p}<$ 0.001 ) in favor of HYADD4 ${ }^{\circledR}$ was recorded at day 14 and maintained at all the follow-up assessments. Data on knee functionality were in line with the VAS pain assessment results. A significant reduction in length and depth of the meniscal lesion, assessed using MRI, was found in the HYADD $4{ }^{\circledR}$ group compared to the control group $(p<0.001)$.

Conclusions: the results of this study may indicate a new treatment option in the conservative management of patients complaining of pain due to meniscal tears. The MRI data suggest that the hydrogel formulation of HA used in this study may also play a role in the healing process of the lesion.

\section{Corresponding Author:}

Nicola Giordan, Ph.D.

Department of Clinical Operations, Fidia Farmaceutici SpA. via Ponte della Fabbrica 3/A, 35031 Abano Terme (PD), Italy

E-mail: ngiordan@fidiapharma.it
Level of evidence: Level I, prospective randomized clinical trial.

Keywords: healing, hyaluronic acid, meniscal tear, magnetic resonance.

\section{Introduction}

Meniscal injuries are the second most common injury to the knee with an incidence ranging from 12 to $14 \%$ and a prevalence of 61 cases per 100,000 persons with a male predominance (1). These injuries can be divided into two groups: traumatic tears and degenerative tears. Traumatic tears occur due to a shear force between the femur and the tibia; they are more frequent in younger patients in whom they are likely to be consequent to an acute traumatic event. Degenerative changes are more frequently observed in older patients (2) and are thought to progress from intrasubstance degeneration within the menisci (3). When articular cartilage is exposed to compression forces, due to degeneration or joint trauma, the lubricating function of the synovial fluid is severely impaired; indeed, the fluid may be diluted due to intra-articular (IA) bleeding or plasma extravasation and this results in a reduced concentration of hyaluronic acid (HA) and lubricin, which are the main joint lubricants (4). Although numerous studies have evaluated the effectiveness of operative treatment of meniscal tears (57 ), the effectiveness of non-operative treatments has received little attention in the literature $(8,9)$.

Rimington et al. (9) provided evidence of the effectiveness of a non-operative protocol of treatment for degenerative medial meniscal tears based on the use of non-steroidal anti-inflammatory drugs (NSAIDs). However, no studies have specifically set out to eva- 
luate the effectiveness of $\mathrm{HA}$ in the treatment of meniscus tears.

We designed a prospective randomized study to investigate the efficacy of IA administration of a hydrogel formulation obtained from a HA derivative in the management of patients with a diagnosis of meniscal tear.

\section{Methods}

\section{Study design}

This observer-blind, parallel-group clinical study was designed to assess the efficacy of IA injections of Hymovis (Fidia Farmaceutici SpA, Italy), a new hydrogel based on a non-crosslinked HA alkylamide (HYADD4 ${ }^{\circledR}$; Fidia Farmaceutici SpA), in patients suffering from meniscal tears. The control group consisted of patients receiving only non-operative treatment. The study was approved by the local ethics committee ("Ethics Committee for Good Clinical Practice", Sacro Cuore Don Calabria Hospital, Negrar-VR, Italy) and was conducted in accordance with the Helsinki Declaration and in compliance with the principles of good clinical practice. Ethics Committee approved written consent was obtained from all the patients before their inclusion in the study. After treatment, the patients were followed up for a minimum of 30 days, a period that could be extended to 60 days if the patient was not referred for surgery at the 30-day evaluation.

\section{Participants}

Male or female patients $(n=50)$ between 18 and 50 years of age with a documented diagnosis of a single meniscal tear (horizontal, longitudinal, flap, radial) were recruited.

Study participants were evaluated clinically and radiographically. Radiographic evaluation, consisting of a weight-bearing anteroposterior view of both knees, a weight-bearing flexed-knee posteroanterior view of both knees, a lateral view, and an axial view of the affected knee, was performed to rule out osteoarthritis (OA). The Kellgren-Lawrence grading system was used to score knee OA (10). The cartilage of the weight-bearing area was graded by two experienced orthopaedic surgeons using the International Cartilage Repair Society (ICRS) grading system (11). All patients enrolled in the study underwent an MRI evaluation. Patients were included in the study only if they had an ICRS Grade 1 lesion in the weight-bearing area and no X-ray or MRI evidence of OA.

Exclusion criteria were: the presence of concomitant pathologies that could interfere with the subject's ability to perform the study or that could confound the study results (e.g. rheumatoid arthritis, metabolic bone disease, gout, Paget's disease, symptomatic chondrocalcinosis, etc.), known or suspected allergic reactions to hyaluronic preparations, arthroplasty of the target knee at any time or any other previous surgery on the target knee within 12 months prior to inclusion, or surgery scheduled during the study period, known or suspected infection of the affected joint, skin conditions of the affected knee such as dermatitis or psoriasis; poor general health, and inability of the patient to comply with the protocol for the entire duration of the study.

\section{Interventions}

At inclusion, patients were randomly assigned (1:1) to two groups receiving the following treatments: the control group (conservative treatment) or the HYADD4® group (HYADD4® plus conservative treatment). The randomization list was produced using a free online randomization plan generator available at: http://www.random.org.

HYADD4® is a class III CE certified medical device already approved for the improvement of joint mobility through the enhancement of synovial fluid viscoelasticity.

The non-operative treatment consisted of two weeks of ice applications, rest and knee off-loading combined with the intake of paracetamol as per patient's needs. Participants had to agree not to use any NSAIDs besides acetaminophen (up to 1,000 $\mathrm{mg} 4$ times per day as needed), and had to discontinue the use of acetaminophen at least 24 hours before each office visit for the duration of the study.

Patients allocated to the HYADD ${ }^{\circledR} 4$ group received two IA injections of HYADD $4^{\circledR}$ two weeks apart in addition to the conservative treatment.

\section{Outcome measures}

Clinical evaluations were performed at baseline and after 14 and 30 days. Patients who, at 30 days, were not referred for knee surgery were requested to undergo a further evaluation 60 days after the beginning of treatment. The evaluations could be performed within a time window of \pm 2 days. 
Clinical outcome, i.e. pain reduction and improvement in knee functionality, was assessed through pain measurement and administration of the Western Ontario and McMaster Universities Osteoarthritis Index (WOMAC) questionnaire. Patient's health status was investigated using the SF-36 quality of life questionnaire and considering the patient's and investigator's global assessment.

Pain perceived by the patient was measured using a visual analog scale (VAS) consisting of a line ranging from $0 \mathrm{~mm}$, indicating the absence of pain, to 100 $\mathrm{mm}$ indicating unbearable pain. The patients, asked to indicate their perceived pain on the VAS, were instructed to respond with reference to their pain "at the present time".

The WOMAC questionnaire consists of three subscales: the WOMAC pain scale (Section A), made up of five questions, the WOMAC stiffness scale (Section B) comprising two questions, and the WOMAC physical function scale (Section C), made up of 17 questions. The patient's response to each of the 24 questions was measured on a five-point Likert scale with higher scores indicating greater symptom severity $(0=$ none, $1=$ slight, $2=$ moderate, $3=$ severe and $4=$ extreme).

The SF-36 questionnaire is designed to measure two dimensions: physical functioning and mental health. Scores for each domain range from 0 to 100, with high scores indicating a better status.

The patient global assessment $(\mathrm{PtGA})$ and clinical observer global assessment (COGA) on how the treated knee affected the patient's health status were obtained by means of a VAS: both the patient and the investigator made their global assessment on a $0-100 \mathrm{~mm}$ VAS where 0 corresponded to "not at all" and 100 to "extremely".

Local tolerability at the site of injection, in the HYADD4® group, was assessed considering redness and pain occurring in the minutes following treatment sessions; systemic adverse events were recorded throughout the duration of the study.

Assessment of length and depth of meniscal tears was performed by MRI at baseline, day 30 and day 60 (when applicable). Since the MRI assessment of meniscal tears was a secondary study endpoint, the investigators recorded only the presence or absence of a reduction in length and depth of the lesion (comparing MR images against baseline) and not the measurements.

Knee MR images were evaluated by a radiologist who assessed the length and depth of the meniscal tears and by an orthopaedic surgeon who evaluated the images to exclude, as per the exclusion criteria, the presence of concomitant pathologies that could interfere with the subject's ability to perform the study, or that could confound the study results. Both evaluators were blinded to the patients' data.

T1 and T2 sagittal and coronal proton density-weighted images were obtained using a Siemens Avanto 1.5T MRI system (Siemens, Erlangen, Germany). The presence or absence of a tear was based on the presence or absence of a linear signal, brighter than the dark meniscus. The following grading system was used to describe abnormal intrameniscal signals: Grade 1 the signal is oval or globular in appearance and does not communicate with any meniscal surface; Grade 2 - the signal is a more linear but, similarly, does not communicate with the articular surfaces; Grade 3 the signal, located within the meniscus, is linear and should communicate with either the superior or the inferior articular surfaces. Grade 1 and 2 signals are consistent with intrasubstance myxoid degeneration, whereas a Grade 3 signal is consistent with a tear (12). At baseline, only a Grade 3 signal was taken as evidence of a meniscal tear (13) and tears were classified according to length, depth and tear pattern.

Tear length was assessed as the length of a meniscal tear that reaches the surface of the meniscus. Tear depth was rated from Grade 1 to Grade 3 according to the MRI classification.

At baseline, tear patterns were classified as longitudinal (located anywhere along the meniscus), horizontal (begins at the inner margin of the meniscus and extends towards the capsule), radial (begins at the inner margin and extends towards the capsule) or flap (either a vertical flap tear or a horizontal flap tear).

By comparing the follow-up MR images with the baseline images, the presence or absence of a reduction in length and depth of the lesion was identified. The occurrence of a new lesion with respect to baseline was considered a worsening and recorded as an adverse event. Reductions in the length and depth of the meniscal tear subsequent to baseline, assessed radiologically, were measured in relation to the tear evaluated at study entry.

\section{Data analysis}

A sample size of 50 patients was established conside- 
ring an $\alpha=0.05$, a power of $90 \%$, a significance test on repeated continuous measures, two treatment groups and an effect size of 0.37 (small-medium effect size).

All data were stored and analyzed using the SPSS statistical package 10.0 (SPSS Inc., Chicago, Illinois, USA). The full analysis set, which included all patients receiving treatment (at least conservative treatment), was considered for the statistical analysis performed on absolute values or on changes from baseline, as applicable. In the event of missing values and prematurely discontinued patients, the last observation carry forward method was used before performing inferential analysis.

To calculate the total and partial WOMAC scores the indications provided in the User Guide IX of the WOMAC Osteoarthritis Index were followed; in the event of missing data the average value of the subscale (pain, stiffness or physical functioning) was substituted in lieu of the missing item value(s).

In order to normalize the WOMAC index to a $0-100$ scale all single scores in each subscale were summed, multiplied by 100 and divided by the possible maximum score for the relevant subscale. A total score was created by combining the three subscales and, again, the total score was converted into a normalized score out of 100 as described in the WOMAC user's handbook.

The physical functioning score and the mental health score of the SF-36 questionnaire were calculated following the guidelines of the instrument.

The following descriptive statistics were produced at each study time point for each variable collected: mean, median, standard deviation, minimum and maximum were calculated for continuous variables, number and percentage of patients in each category for categorical data.

Differences between treatment groups were analyzed by a multiple analysis of variance (MANOVA) for paired data if assumptions for the test were satisfied, and, in the event of significance, comparisons against baseline were performed. Alternatively, non-parametric tests (Fisher's and Wilcoxon test) were conducted. All $\mathrm{p}$ values derived from two-sided statistical tests and $\mathrm{p}$-values $<0.05$ were considered statistically significant. If necessary, adjustment of the $\alpha$ level was carried out by Bonferroni correction.

\section{Results}

Fifty outpatient subjects were enrolled into the study in less than three months (January-March 2012) and randomized to one of the treatment groups: 25 patients were allocated to the HYADD $4{ }^{\circledR}$ group and 25 to the control group.

All the patients receiving HYADD4 ${ }^{\circledR}$ completed the study, while only 17 of the 25 patients in the control group attended the 60-day end-of-study evaluation (Fig. 1): one patient was lost to follow-up and seven patients discontinued the study as they were not responsive to treatment.

No major protocol deviations emerged from data: all the enrolled subjects met the inclusion and exclusion criteria and visits were performed at acceptable intervals, with some exceptions: 5 patients in the HYADD $4{ }^{\circledR}$ group underwent the 30 -day evaluation with a delay of 13 days and attended the 60-day evaluation 10 days after the due date; 1 patient in the control group underwent the 60-day evaluation 12 days in advance. All 25 patients allocated to the HYADD $4^{\circledR}$

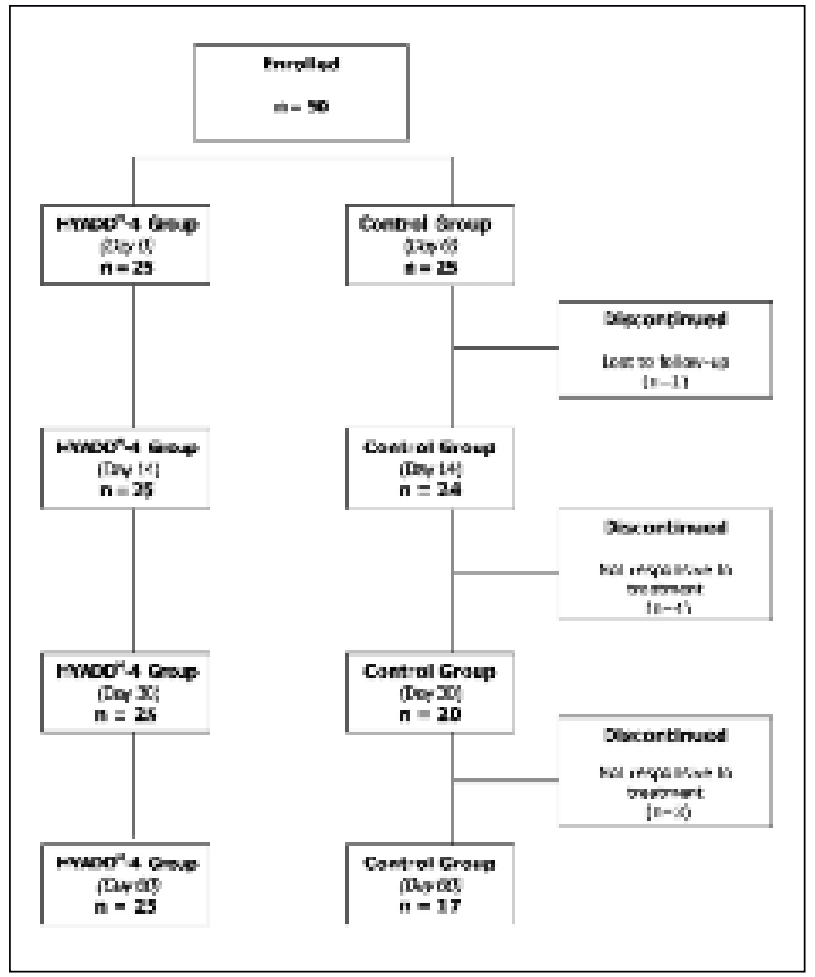

Fig. 1. Flow diagram of the study. 


\section{oincts}

.

group received the two injections two weeks apart as planned.

Table 1 summarizes the main baseline characteristics of the 50 patients. All the patients were in good general health. No significant concomitant diseases or concurrent therapies were recorded.

In both groups, an improvement in pain perceived by the patient was shown from baseline up to the 60-day evaluation. The ANOVA for repeated measures conducted on VAS pain revealed a treatment effect: the pain perceived by the patients significantly diminished over time but the reduction observed in patients treated with HYADD $4^{\circledR}$ was greater than that observed in patients belonging to the control group $(\mathrm{p}<0.001)$. The reduction was recorded at the 14-day evaluation and maintained at all the subsequent evaluations (Fig. 2).

Data on knee functionality, evaluated by the WOMAC subscores and total score, were in line with the VAS pain results. All the subscores and the total score decreased over time and the mean value of the scores in the HYADD $4^{\circledR}$ group was always lower than the mean value observed in the control group.

When considering changes from baseline in the subscores or total score (Tab. 2), the Wilcoxon test con-

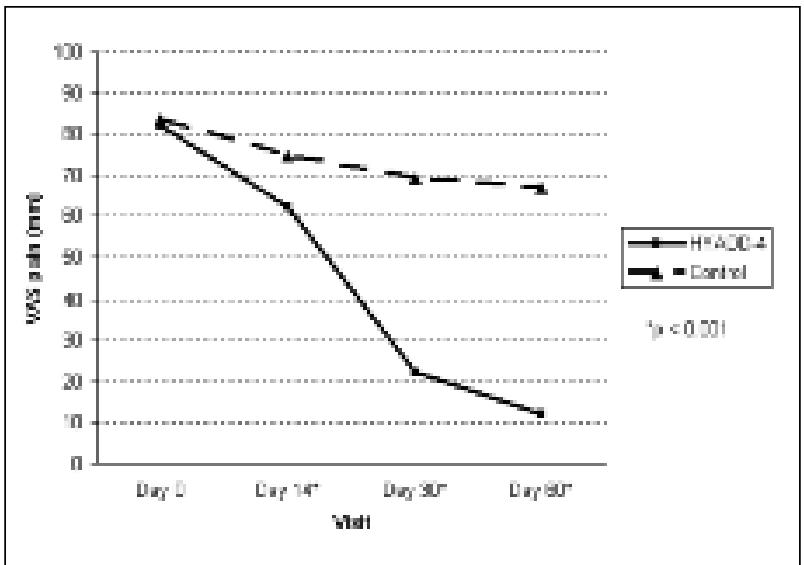

Fig. 2. Reduction of pain during the study as measured using VAS $(\mathrm{mm})$ by the treatment group.

Table 1. Baseline characteristics of 50 patients with meniscal lesions allocated to the HYADD4 ${ }^{\circledR}$ group or the control group*.

\begin{tabular}{|c|c|c|}
\hline & $\begin{array}{c}\text { HYADD4 }^{\circledR} \text { Group } \\
(\mathrm{n}=25)\end{array}$ & $\begin{array}{c}\text { Control Group } \\
(\mathrm{n}=25)\end{array}$ \\
\hline Age, years & $30 \pm 4.4$ & $33 \pm 3.9$ \\
\hline Sex, $\%$ male & 64 & 48 \\
\hline Weight, kg & $76 \pm 12.5$ & $72 \pm 9.7$ \\
\hline Height, $\mathrm{cm}$ & $179 \pm 8.9$ & $175 \pm 8.2$ \\
\hline \multicolumn{3}{|l|}{ Smoking habits: } \\
\hline - never smoked, \% & 56 & 60 \\
\hline - current smoker, \% & 28 & 20 \\
\hline - ex-smoker, \% & 16 & 20 \\
\hline Affected knee, $\%$ right & 60 & 52 \\
\hline Use of assistive device, $\%$ & 16 & 28 \\
\hline ICRS grade $1, \%$ & 100 & 100 \\
\hline \multicolumn{3}{|l|}{ MRI meniscal lesion type: } \\
\hline - Longitudinal, \% & 32 & 36 \\
\hline - Horizontal, \% & 48 & 24 \\
\hline - Flap, \% & 4 & 12 \\
\hline - Radial, \% & 16 & 28 \\
\hline Pain VAS, mm & $82.0 \pm 9.13$ & $83.6 \pm 7.82$ \\
\hline WOMAC pain score & $62.8 \pm 12.59$ & $70.0 \pm 13.84$ \\
\hline WOMAC stiffness score & $66.0 \pm 14.67$ & $80.5 \pm 11.46$ \\
\hline WOMAC physical functioning score & $62.8 \pm 6.99$ & $73.1 \pm 8.64$ \\
\hline WOMAC global score & $63.1 \pm 7.86$ & $73.1 \pm 8.85$ \\
\hline SF-36 physical functioning score & $28.1 \pm 3.46$ & $27.6 \pm 3.76$ \\
\hline SF-36 mental health score & $44.3 \pm 5.03$ & $41.4 \pm 8.07$ \\
\hline Patient global assessment & $77.6 \pm 21.90$ & $87.2 \pm 11.28$ \\
\hline Physician global assessment & $80.5 \pm 7.11$ & $83.8 \pm 7.94$ \\
\hline
\end{tabular}

* Except where indicated otherwise, values are the mean \pm standard deviation. 
ducted at each time point showed a greater decrease in the HYADD4 ${ }^{\circledR}$ group with respect to the control group $(p<0.017)$ for each subscore and for the total score.

It is worth mentioning that as regards the WOMAC subscores for stiffness and for physical function and the WOMAC total score, a non-homogeneity between the treatment groups was observed at baseline. These differences are the reason for the use of a non-parametric approach for paired data (Wilcoxon test) to analyze changes from baseline values and the adaptation of the $\alpha$ level according to the Bonferroni's method. However, even though randomization procedures failed to guarantee homogeneity between treatment groups for each study parameter at baseline, the primary endpoint (VAS and WOMAC pain subscore) baseline data confirmed homogeneity between the two groups.

At baseline, the patients allocated to the two treatment arms were similar in terms of both the physical functioning score and the mental health score on the SF36 questionnaire. In patients treated with HYADD $4{ }^{\circledR}$, the physical functioning score increased from a mean value of $28.1 \pm 3.46$ at baseline to $48.5 \pm 4.27$ at 30 days and to $54.6 \pm 5.43$ at the 60-day evaluation; patients allocated to the control group presented a mean value at baseline of $27.6 \pm 3.76$, which rose to $33.1 \pm 8.45$ at day 30 and $37.1 \pm 10.0$ at the 60 -day evaluation.

As regards the mental health score, in patients treated with HYADD $4^{\circledR}$ the mean value of $44.3 \pm 5.03$ recorded at baseline increased to $56.3 \pm 4.21$ at 30 days and $56.6 \pm 4.25$ at the 60 -day evaluation; instead, in the control group the mean values were $41.4 \pm 8.07$ at baseline, $44.7 \pm 12.28$ at 30 days, and $43.2 \pm 11.41$ at 60 days.

While the SF-36 physical functioning score increased significantly between baseline and the end of the study and the ANOVA for repeated measures $(p<0.001)$ showed a treatment effect in favor of HYADD $4{ }^{\circledR}$ at all assessments, the mental health score did not change significantly between baseline and the 30-day evaluation. The ANOVA for repeated measures $(\mathrm{p}<0.001)$ showed a treatment effect in favor of HYADD $4{ }^{\circledR}$ at the 60 -day evaluations.

The PtGA and COGA on how the treated knee affected the patient's status were evaluated by means of a VAS; both assessments revealed an improvement over time. The ANOVA for repeated measures on PtGA showed a statistically significant difference versus baseline at each study evaluation and between treatment groups $(\mathrm{p}<0.001)$ in favor of HYADD $4^{\circledR}$. The COGA VAS values were not normally distributed (Kolmogorov-Smirnov test), therefore a MannWhitney test was used to compare treatment groups at each evaluation and the $\alpha$ level was corrected according to Bonferroni. At each evaluation a statistically significant difference between groups was observed $(p<0.017)$ in favor of HYADD4 ${ }^{\circledR}$.

The reduction of length and depth of the meniscal lesion, detected by the MRI, was particularly evident in the HYADD $4{ }^{\circledR}$ group $(80 \%$ of patients improving at the third and $92 \%$ at the fourth evaluation) versus $16 \%$ and $20 \%$ of patients in the control group $(\mathrm{p}<0.001$ on Fisher's exact test) (Fig. 3).

With the exception of slight pain at the injection site that resolved spontaneously within a few minutes, no patient treated with HYADD $4{ }^{\circledR}$ complained of significant adverse effects of the device either in the minutes following treatment or at any time during the study. During the study a total of 10 adverse events (two in the HYADD $4{ }^{\circledR}$ group and eight in the control group) were reported in eight patients. All adverse events were related to the knee condition and consisted of a

Table 2. Changes from baseline in WOMAC sub-scores and total score.

\begin{tabular}{|c|c|c|c|c|c|c|}
\hline & \multicolumn{2}{|c|}{ Day 14} & \multicolumn{2}{|c|}{ Day 30} & \multicolumn{2}{|c|}{ Day 60} \\
\hline & $\begin{array}{c}\text { HYADD4 }^{\circledR} \\
\text { (mean } \pm \text { s.d.) }\end{array}$ & $\begin{array}{c}\text { Control } \\
\text { (mean } \pm \text { s.d.) }\end{array}$ & $\begin{array}{l}\text { HYADD4 }^{\circledR} \\
\text { (mean } \pm \text { s.d.) }\end{array}$ & $\begin{array}{c}\text { Control } \\
(\text { mean } \pm \text { s.d.) }\end{array}$ & $\begin{array}{l}\text { HYADD4 }^{\circledR} \\
\text { (mean } \pm \text { s.d.) }\end{array}$ & $\begin{array}{c}\text { Control } \\
\text { (mean } \pm \text { s.d.) }\end{array}$ \\
\hline Pain & $-23.6 \pm 11.32$ & $10.8 \pm 10.28$ & $-46.8 \pm 8.77$ & $-23.0 \pm 21.94$ & $-57.6 \pm 10.62$ & $-26.6 \pm 20.09$ \\
\hline Stiffness & $-24.5 \pm 20.88$ & $-14.0 \pm 11.59$ & $-44.5 \pm 21.97$ & $-25.9 \pm 13.59$ & $-59.5 \pm 13.64$ & $-31.4 \pm 19.59$ \\
\hline Physical & $-23.8 \pm 9.52$ & $-11.5 \pm 5.58$ & $-48.7 \pm 11.14$ & $-21.1 \pm 15.19$ & $-58.1 \pm 4.12$ & $-27.4 \pm 18.84$ \\
\hline Total & $-23.8 \pm 9.00$ & $-11.6 \pm 5.37$ & $-48.0 \pm 9.9$ & $-21.9 \pm 15.58$ & $-58.1 \pm 4.53$ & $-27.6 \pm 18.25$ \\
\hline
\end{tabular}




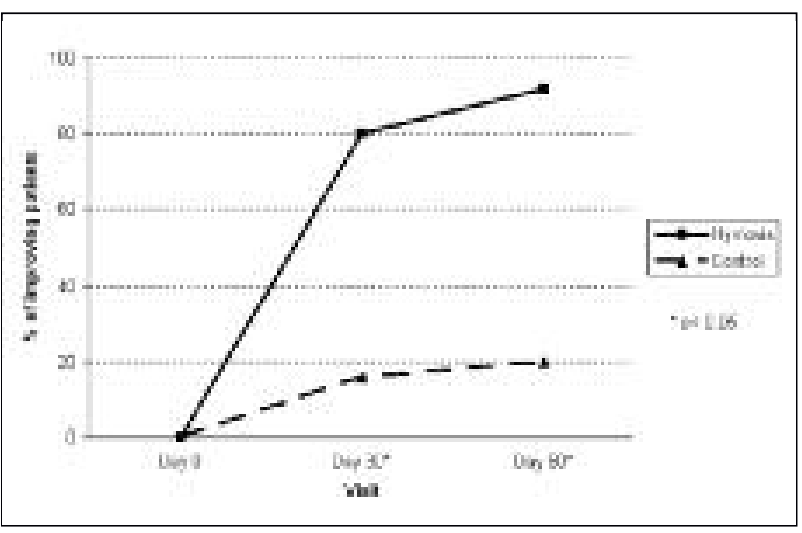

Fig. 3. Reduction of length and depth of the meniscal lesion: $\%$ of improving patients by follow-up assessments and treatment group.

worsening of the disease or the occurrence of a new lesion detected by MRI: one patient (4\%) in the HYADD $4^{\circledR}$ group developed a new lesion versus three $(12 \%)$ occurrences of new lesions in the control group. In particular, in two cases the development of a new lesion did not correspond to an increase in pain or to a reduction in knee functionality as resulting from the WOMAC questionnaire.

\section{Discussion}

As the menisci are fundamental structures for the maintenance of knee homeostasis, playing a key role in knee biomechanics, meniscal tissue should be preserved whenever possible and partial or total meniscectomy should be left as the final option. Indeed, long-term follow-up studies have shown that virtually all meniscectomized knees develop arthritic changes over time (14); therefore, when considering treatment options for a patient complaining of pain due to a meniscal tear, conservative options should be considered as a first-line therapy before surgical treatment. This study was conducted with the purpose of evaluating a non-operative treatment protocol designed to reduce pain and improve lesion healing in patients with degenerative meniscal tears. It prospectively compared two injections of HYADD $4^{\circledR}$ (administered two weeks apart) plus non-operative treatment versus non-operative treatment alone. The study results indicated that IA HYADD $4{ }^{\circledR}$ may be effective in the conservative management of pain related to meniscal tears and may also play a role in the healing process of the lesions.
Patients receiving HYADD $4^{\circledR}$ reported a higher reduction in pain and a greater improvement of knee functionality with respect to patients receiving only conservative therapy. The ameliorative effect of HYADD $4{ }^{\circledR}$ was already evident after the second injection (14 days from baseline) and was maintained for up to 60 days, when the study terminated.

Patient and investigator assessments on how the treated knee affects the patient's health status confirm the results obtained with regard to pain, as the impact of the disease was considered by both investigator and patient to be significantly reduced from the day 14 evaluation onwards. The SF-36 physical functioning score showed a better improvement in patients receiving the study treatment, as did the SF-36 mental health score. A potential role of HYADD $4{ }^{\circledR}$ in promoting meniscal lesion healing was also investigated by MRI assessments and a reduction in length and depth of the meniscal lesion was observed in a higher percentage of patients receiving $\mathrm{HYADD} 4^{\circledR}$ with respect to patients treated by conservative therapy alone. Indeed, HA presents healing properties that could help to heal the damaged tissue itself.

Despite the breadth of potential indications for IA injections of HA in the knee joint, few studies have investigated use of this treatment in knee lesions other than OA, such as meniscal tears $(15,16)$.

The mechanisms of meniscal healing follow two patterns: the extrinsic and the intrinsic pathway. The extrinsic pathway, occurring mainly in the vascular area of the lesion, is based on the network of capillaries that supply the lesion with undifferentiated mesenchymal cells as well as nutrients that induce healing. The intrinsic pathway is based on the self-repair capacity of the meniscal fibrocartilage and the synovial fluid (14). Although, to date, no substance has been found to induce predictable, spontaneous meniscal healing, a recent in vitro study conducted on human synovial cells stimulated with HA (17) showed that treatment with HA $(0.1 \mathrm{mg} / \mathrm{mL})$ increases the expression of TGF- $\beta 1$ and VEGF. The possibility of increasing levels of these growth factors in the injured meniscus may explain the mechanism of action of HYADD $4{ }^{\circledR}$ and may open up new treatment options. The rationale of using hyaluronans on meniscal tears is supported by several studies conducted in animals and humans. Specifically, preclinical studies demonstrated the efficacy of IA injections of HYADD $4{ }^{\circledR}$ in 
the induction of gait improvement in animals with bilateral lateral meniscectomy (18) and in the antinociceptive activity of the treatment in the first week after partial medial meniscectomy in guinea pigs (19). Furthermore, in pathological human chondrocytes and synoviocytes stimulated by IL- $1 \beta$, HYADD $4^{\circledR}$ was able to reduce the gene expression of degradative enzymes and inflammatory cytokines (20).

HA preparations in human studies have been reported to improve symptoms and rating scores and decrease the use of NSAIDs in patients who underwent partial meniscectomy $(16,21)$. Considering that the half-life of native HAs in the joint could be approximately one to two days (22), the use of this modified HA-based product with a slow enzymatic and free-radical degradation and prolonged half-life should result in an extended residence time with a consequent prolongation of the related clinical benefits (23).

It is unlikely that the observed sustained beneficial effect on pain associated with meniscal tears is related only to a temporary restoration of the synovial fluid lubrication and viscoelasticity, in accordance with the viscosupplementation rationale; rather, it is more plausible that the benefits of HA therapy are due to both mechanical and biological activities (24). The exact mechanism of action of HAs are unknown, but the positive influence of IA administration of $\mathrm{HA}$ on several biological processes involved in joint homeostasis has been demonstrated, and may explain the sustained clinical effects. Hyaluronans can induce the synthesis of endogenous HA by synovial cells, stimulate chondrocyte proliferation, and inhibit cartilage degradation (chondroprotection) (25). In a recent study, Greenberg et al. (26) demonstrated an elevated glycosaminoglycan content in HA-treated cartilage tissue and the conclusions of their study support a biosynthetic-chondroprotective mechanism (26). Other studies demonstrated that stimulation of the synthesis of HA and extracellular matrix components by synovial fibroblasts and inhibition of leukocyte migration, chemotaxis, and adhesion contribute to observed antiinflammatory effects (27).

In several studies treatment with sodium hyaluronate has been shown to increase the viscoelastic properties of the synovial fluid and repair the articular cartilage by improving cellular metabolism (28).

Studies on animal models indicate that significant, structural meniscal healing occurs within a short time frame. Two preclinical studies have been performed in rabbits with meniscal lesions $(29,30)$. In the study by Ishima et al. (29) the meniscal lesion was obtained by creating a longitudinal tear in the peripheral region of the medial meniscus, while in that of Suzuki et al. (30) the meniscal lesion was made by creating a cylindrical defect on the lateral meniscus. In both studies weekly IA injections of HA or saline were performed.

Suzuki et al. evaluated the effect of HA treatment at one and six weeks after the start of a series of weekly injections and the treatment was compared with injections of phosphate buffer. HA administration caused a significant increase in the rate of filling of the defect. The cell population in the repair tissue changed from fibroblast-like cells to chondrocyte-like cells as the repair progressed. At six weeks, the ratio of chondrocyte-like cells to all cells was higher than that of the control and the authors concluded that HA increases the healing rate of meniscal injury (30).

In Ishima's study the effect on meniscal tears of HA treatment (five weekly injections, starting one week after surgery) was evaluated by histology at 6 and 12 weeks after surgery. On a general morphology examination, there was evidence of meniscal healing in both groups, but the healing rate of the HA group was significantly higher than that of the saline-injected group. Histologically, meniscal healing was evident at 6 weeks after surgery in both groups (29).

These two studies confirm that in animals meniscal healing is possible, that hyaluronan injections increase the healing rate, and that meniscal healing is evident just a few weeks after the lesion.

In humans, as well described by Weiss et al. (31), the meniscus has a greater potential to heal and the healing potential depends on the location, type, length and stability of the tear. In the Author's opinion this explained the degree of healing also seen in the control group. In the literature, Han et al. (32) reported a case of spontaneous healing of a displaced buckethandle tear. This patient had an unstable displaced bucket-handle tear of the lateral meniscus in a stable knee, which healed spontaneously within six weeks without surgery. Six weeks after the diagnosis, the patient was no longer complaining of pain and had regained a full range of motion. The final follow-up MRI study showed reduction of the torn meniscal fragment without any signal changes suggestive of a meniscal tear. Thus, it is possible that IA HA con- 
tributes to the spontaneous healing of the meniscus providing the knee joint with a supply of HA that may be beneficial to the healing process. However, the short time frame for meniscus healing observed in animals remains to be confirmed in humans.

Although this study may answer some questions on

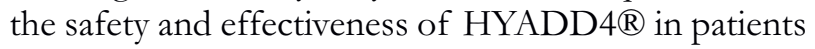
affected by meniscal tears, the relationship between the biological and clinical actions of HA remains to be explored. The strengths of the therapy proposed in this study include its simplicity and the safety of the product used. Moreover, thanks to its high viscosity and elasticity (33), together with its prolonged residence time, HYADD4® is able to relieve pain and the treatment seems to improve lesion healing with a short treatment regimen. The limitations of this study include the small sample size, the short follow-up and the absence of recording of concomitant NSAID intake. The small number of patients enrolled in the study and the short follow-up limited the power of the results even though a large statistical difference between the two groups was found, especially in pain reduction and lesion healing.

The long-term efficacy of HYADD $4{ }^{\circledR}$ remains to be explored and it could be interesting to perform a pharmacoeconomic study to investigate the cost and effectiveness of this treatment protocol compared to the standard conservative therapy. High-quality viscosupplements can sometimes be expensive, but a cost-benefit analysis may show that this treatment is able to reduce pain and NSAID intake, delaying meniscectomy in patients with meniscal lesions; it may thus constitute a less invasive, short-regimen treatment that will finally represent a more cost-effective option.

In conclusion, IA of the new hydrogel evaluated in this study seems to satisfy an unmet need: the introduction of HYADD $4{ }^{\circledR}$ seems to restore the physiological environment of the joint and, while reducing pain, is likely to promote the healing process.

\section{Conflict of interest}

This study was an investigator initiated study carried out in accordance with internationally recognized standards, such as the Declaration of Helsinki and the ICH's Guideline for Good Clinical Practice.

The Authors, declare that they have no conflict of commercial interest. Nicola Giordan is an employee of Fidia Farmaceutici SpA. All the Authors state, however, that Fidia Farmaceutici SpA did not solicit this research project or protocols with investigators or institutions. Fidia Farmaceutici SpA was not responsible for the initiation of the study and did not participate in the scientific design of the trial, in the creation of the protocol, management of the study, data analysis, reporting and in the decision to submit the manuscript for publication.

\section{References}

1. Majewski M, Susanne H, Klaus S. Epidemiology of athletic knee injuries. A 10-year study. Knee. 2006;13:184-148.

2. Snoeker BA, Bakker EW, Kegel CA, et al. Risk factors for meniscal tears: a systematic review including meta-analysis. J Orthop Sports Phys Ther. 2013;43:352-367.

3. Metcalf $\mathrm{MH}$, Barrett GR. Prospective evaluation of 1485 meniscal tear patterns in patients with stable knees. Am J Sports Med. 2004;32:675-680.

4. Elsaid KA, Fleming BC, Oksendhal HL et al. Decreased lubrici concentrations and markers of joint inflammation in the synovial fluid of patients with anterior cruciate ligament injury. Arthritis Rheum. 2008;58:1707-1715.

5. Bonamo JJ, Kessler KJ, Noah J. Arthroscopic meniscectomy in patients over the age of 40. Am J Sports Med. 1992;20:422-428.

6. Fabricant PD, Jokl P. Surgical outcomes after arthroscopic partial meniscectomy. J Am Acad Orthop Surg. 2007;15:647653.

7. Howell JR, Handoll HH. Surgical treatment for meniscal injuries of the knee in adults. Cochrane Database Syst Rev. 2000;2:CD001353.

8. Herrlin S, Hållander M, Wange $\mathrm{P}$, et al. Arthroscopic or conservative treatment of degenerative medial meniscal tears: a prospective randomised trial. Knee Surg Sports Traumatol Arthrosc. 2007;15:393-401.

9. Rimington T, Mallik K, Evans D, et al. A prospective study of the nonoperative treatment of degenerative meniscus tears. Orthopedics. 2009;32. doi: 10.3928/01477447-20090624-06.

10. Kellgren JH, Lawrence JS. Radiological assessment of osteoarthrosis. Ann Rheum Dis. 1957;16:494-502.

11. Kleemann RU, Krocker D, Cedraro A, et al. Altered cartilage mechanics and histology in knee osteoarthritis: relation to clinical assessment (ICRS Grade). Osteoarthritis Cartilage. 2005;13:958-963.

12. Mink JH, Reicher MA, Crues JV. Magnetic Resonance Imaging of the Knee. Raven Press, New York, NY, 1987.

13. Behairy NH, Dorgham MA, Khaled SA. Accuracy of routine magnetic resonance imaging in meniscal and ligamentous injuries of the knee: comparison with arthroscopy. Int Orthop. 2009;33:961-967.

14. Laasonen EM, Wilppula E. Why a meniscectomy fails. Acta Orthop Scand. 1976;47:672-675.

15. de Albornoz PM, Forriol F. The meniscal healing process. Muscles, Ligaments and Tendons J. 2012;2:10-18.

16. Mathies B. Effects of Viscoseal, a synovial fluid substitute, on recovery after arthroscopic partial meniscectomy and joint lavage. Knee Surg Sports Traumatol Arthrosc. 2006;14:32-39. 
17. Lee YT, Shao HJ, Wang JH, et al. Hyaluronic acid modulates gene expression of connective tissue growth factor (CTGF), Transforming growth factor-beta1 (TGF-b1), and vascular endothelial growth factor (VEGF) in human fibroblast-like synovial cells from advanced-stage osteoarthritis in vitro. J Orthop Res. 2010;28:492-496.

18. Cake M, Read R, Edwards S, et al. Changes in gait after bilateral meniscectomy in sheep effect of two hyaluronan preparations. J Orthop Sci. 2008;13:514-523.

19. Gomis A, Miralles A, Schmidt RF, et al. Intra-articular injections of hyaluronan solutions of different elastoviscosity reduce nociceptive nerve activity in a model of osteoarthritic knee joint of guinea pig. Osteoarthritis Cartilage. 2009; 17:798-804.

20. Smith MM, Russell AK, Schiavinato A, et al. A hexadecylamide derivative of hyaluronan (HYMOVIS $($ ) has superior beneficial effects on human osteoarthritic chondrocytes and synoviocytes than unmodified hyaluronan. J Inflamm (Lond). 2013;10:26.

21. Hempfling H. Intra-articular hyaluronic acid after knee arthroscopy: a two-year study. Knee Surg Sports Traumatol Arthrosc. 2007;15:537-546.

22. Lindenhayn K, Heilmann $\mathrm{HH}$, Niederhausen $\mathrm{T}$, et al Elimination of tritium-labelled hyaluronic acid from normal and osteoarthritic rabbit knee joints. Eur J Clin Chem Clin Biochem. 1997;35:355-363.

23. Pavan M, Galesso D, Menon G, et al. Hyaluronan derivatives: Alkyl chain length boosts viscoelastic behavior to depolymerization. Carbohydr Polym. 2013;97:321-326.

24. Goldberg VM, Coutts RD. Pseudoseptic reactions to hylan viscosupplementation: diagnosis and treatment. Clin Orthop Relat Res. 2004;419:130-137.
25. Maneiro E, de Andres MC, Fernández-Sueiro JL, et al. The biological action of hyaluronan on human osteoartritic articular chondrocytes: the importance of molecular weight. Clin Expl Rheumatol. 2004;22:307-312.

26. Greenberg DD, Stoker A, Kane S, et al. Biochemical effects of two different hyaluronic acid products in a co-culture model of osteoarthitis. Osteoarthritis Cartilage. 2006;14:814822.

27. Ghosh P, Guidolin D. Potential mechanism of action of intra-articular hyaluronan therapy in osteoarthritis: are the effects molecular weight dependent? Semin Arthritis Rheum. 2002;32:10-37.

28. Borzacchiello A, Mayol L, Schiavinato A, et al. Effect of hyaluronic acid amide derivative on equine synovial fluid viscoelasticity. J Biomed Mater Res A. 2010;92:1162-1170.

29. Ishima M, Wada Y, Sonoda M, et al. Effects of hyaluronan on the healing of rabbit meniscus injured in the peripheral region. J Orthop Sci. 2000;5:579-584.

30. Suzuki Y, Takeuchi N, Sagehashi Y, et al. Effects of hyaluronic acid on meniscal injury in rabbits. Arch Orthop Trauma Surg. 1998;117:303-306.

31. Weiss CB, Lundberg M, Hamberg P, et al. Non-operative treatment of meniscal tears. J Bone Joint Surg Am. 1989;71:811-822.

32. Han JH, Song JG, Kwon JH, et al. Spontaneous healing of a displaced bucket-handle tear of the lateral meniscus in a child. Knee Surg Relat Res. 2015;27:65-7.

33. Finelli I, Chiessi E, Galesso D, et al. A new viscosupplement based on partially hydrophobic hyaluronic acid: a comparative study. Biorheology. 2011;48:263-275 